\title{
Application of 18-fluorodeoxyglucose as an indicator of symptom severity in a rat model of tic disorder following treatment with traditional Chinese medicine
}

\author{
PENGCHENG ZHU ${ }^{1}$, MIN WU ${ }^{1}$, BITAO MA ${ }^{1}$ and SHUXIA WANG ${ }^{2}$ \\ ${ }^{1}$ Department of Traditional Chinese Medicine, Xinhua Hospital Affiliated to Shanghai Jiao Tong University School \\ of Medicine, Shanghai 200092; ${ }^{2}$ Department of Pediatrics, Yueyang Hospital of Integrated Traditional Chinese and \\ Western Medicine Affiliated to Shanghai University of Traditional Chinese Medicine, Shanghai 200437, P.R. China
}

Received August 8, 2019; Accepted June 24, 2020

DOI: $10.3892 /$ etm.2020.9483

\begin{abstract}
Quantitative evaluation of tic disorders (TDs) is challenging as there are few objective indicators that can be used for the assessment of treatment outcomes. $18 \mathrm{~F}$-Fluorodeoxyglucose (FDG) is a radioactive tracer that is able to cross the blood-brain barrier and can be detected by positron emission tomography/CT (PET/CT). In the present study, it was hypothesized that FDG PET/CT scan can be applied to reflect the severity of tic symptoms in a rat TD model, where signals detected in the brain striatum can be used to evaluate the efficacy of tic treatment with traditional Chinese medicine (TCM). A rat model of TD was established by treatment with iminodipropionitrile. Rats were divided into the following four groups ( $n=10$ each): i) Control; ii) TCM; iii) haloperidol; and iv) model only. Observations of stereotypic behavior in rats were subsequently scored and micro-PET/CT was used to evaluate the rate of FDG uptake. Stereotypy scores were found to be significantly higher $(\mathrm{P}<0.05)$ in the TD rat model $(\mathrm{P}<0.05)$ compared with those in control rats. Both stereotypy scores $(\mathrm{P}<0.05)$ and standardized FDG uptake values (SUV; $\mathrm{P}<0.05$ ) were revealed to be significantly reduced in the TD model rats after treatment with TCM or haloperidol. SUV correlated positively with stereotypy score $(\mathrm{R}=0.926$; $\mathrm{P}<0.05)$ and the SUV scores were found to be significantly different among control group, TCM group, haloperidol group and model only group $(\mathrm{P}<0.05)$. These data suggest that the application of FDG in the striatum can be used to evaluate the effectiveness of TCM treatment for TDs.
\end{abstract}

Correspondence to: Dr Min Wu, Department of Traditional Chinese Medicine, Xinhua Hospital Affiliated to Shanghai Jiao Tong University School of Medicine, 1665 Kongjiang Road, Yangpu, Shanghai 200092, P.R. China

E-mail:wumin@xinhuamed.com.cn

Key words: 18-Fluorodeoxyglucose, brain regeneration, tic disorder

\section{Introduction}

Tic disorders (TDs) constitute a spectrum of heritable neuropsychiatric conditions, including Tourette syndrome and attention deficit hyperactivity disorder, that are characterized by the presence of tics that begin in childhood, typically peaking in severity just before adolescence before improving in adulthood (1). Tics can cause subjective discomfort in the patient such as pain or injury, sustained social problems such as social isolation, bullying; emotional problems such as reactive depressive symptoms and functional interference such as academic underachievement (2). A systematic review and meta-analysis of tic disorders in children in China indicated that the prevalence of TDs was up to $6.1 \%$, which differed based on sex, age and geographical location (3). Many factors hinder the assessment of TD severity, including spontaneous variations in tics in an individual over time, large variability in the impact of a given level of physical tic severity on an individual or their family and the tendency of patients to suppress their tics, especially when in the presence of a clinician (4). Therefore, the evaluation of tics and their associated comorbidities relies upon self-reported scales and clinician-derived interviews (5). However, investigation into TDs using neuroimaging is becoming a popular topic of research, as functional abnormalities in the striatum of the brain are considered to be closely associated with TDs $(6,7)$.

The pathophysiological model of tics generally involves disruptions in $\gamma$-aminobutyric acid transmission (8). The model, which we term the tic generation model (TGM), is based on two kernels that are used for scaling the factors (cortical input and striatal feedback). The model is a simplified version of the simplified spike response model (SRM) that has been used extensively to model neuronal activity (9). Haloperidol, which can effectively inhibit the excitability of the cortical motor area by restraining the activity of dopamine receptors (10), has been approved by the US Food and Drug Administration for the treatment of TDs. In addition, Traditional Chinese medicine (TCM) is also believed by some to be suitable for the treatment of psychiatric disorders (11). A number of studies in China have 
previously suggested that $18 \mathrm{~F}$-Fluorodeoxyglucose (FDG) can be combined with the Yale Global Tic Severity Scale (YGTSS) to evaluate patients with TD $(12,13)$. Therefore, the aim of the present study was to assess the relationship between FDG signals in the striatum of the brain and the stereotypy scores of TD model rats following treatment with either TCM or haloperidol.

\section{Materials and methods}

Rat model. A total of 40 male Sprague-Dawley rats (age, 4 weeks; mean weight, $110 \pm 12$ g; Xinhua Hospital Experimental Animal Center) were housed in an air-conditioned animal room on a 12 -h light/dark cycle at $22 \pm 3^{\circ} \mathrm{C}$ and $50 \pm 10 \%$ humidity. The rats were provided with a laboratory diet and water ad libitum. After 1 week of adaptation, rats were randomly divided into the control $(n=10)$ and experimental $(n=30)$ groups. Rats in the experimental group received daily intraperitoneal (i.p.) injections of iminodipropionitrile (IDPN; Sigma-Aldrich; Merck KGaA) at $150 \mathrm{mg} / \mathrm{kg}$ for 7 days. Rats in the control group were injected with normal saline $(0.9 \% ; 5 \mathrm{ml} / \mathrm{kg}$, i.p.). The relationship between stimulation and behavior was assessed by using peristimulus tic histograms with $0.01 \mathrm{sec}$ bins. To calculate the success percentage in inducing tics, unsuccessful stimuli that were immediately (500 millisec) preceded by a tic were removed from the total number of stimuli in the calculation. After 7 days, the behavior of rats in the experimental group was analyzed and they were further divided into the following three groups ( $n=10$ per group): i) TCM; ii) haloperidol; and iii) model only. Rats in the TCM group received a TCM concoction including $10 \mathrm{~g}$ Tian Ma (Rhizoma Gastrodiae), $10 \mathrm{~g}$ Gou Teng (Ramulus Uncariae cum Uncis), $10 \mathrm{~g}$ Shen Jin Cao (Herba Lycopodii), $5 \mathrm{~g}$ Quan Xie (Scorpio) and $10 \mathrm{~g} \mathrm{Xin}$ Yi (Flos Magnoliae), a mixture of compounds as described to treat tic disorders in a previous study (14). TCM compounds were provided as granules, which were mixed and boiled in $100 \mathrm{ml}$ water for $60 \mathrm{~min}$. All TCM compounds were supplied by Jiangyin Tianjiang Pharmaceutical Co., Ltd. Rats were administered with the TCM compound daily by oral gavage at $9 \mathrm{~g} / \mathrm{kg}$, whilst rats in control group received normal saline and rats in the haloperidol group received haloperidol $(0.1 \mathrm{mg} / \mathrm{ml})$ daily by oral gavage. The intervention lasted for 2 weeks before behavioral analysis and Micro-PEC/CT were conducted for each group. All experimental and animal care procedures were approved by the Ethics Committee of Xinhua Hospital Affiliated to Shanghai Jiaotong University School of Medicine (Shanghai, China). An overview of the experimental procedures is shown in Fig. 1.

Behavioral analysis. Animals were observed during 30-min sessions by two trained observers who were blind to the experimental conditions (7). Observations were made at 5-10 min, 15-20 min and 25-30 min for 1-min periods, for a total of three periods. The items of behavioral stereotypies considered in this analysis were sniffing, focused sniffing and licking/shaking. Several classifications of oral stereotypies, including wood chip eating, self-gnawing, biting, licking that was not associated with grooming and 'taffy pulling' (i.e. repetitive paw-to-mouth movements), were also recorded. A rat that engaged in $\geq 1$ episodes of the same behavior received a score of 1 . Animals that exhibited $\geq 1$ behaviors of any other classification received an additional point for each stereotypy observed. Scores ranged from 0-5 and were averaged for each experimental group. Higher scores indicated increasing severity.

Micro-positron emission tomography/CT (PET/CT). Micro-PET/CT (Siemens Inveon MM PET/CT scanner; Siemens AG) was conducted at Ruijin Hospital Affiliated to Shanghai Jiao Tong University School of Medicine (Shanghai, China). All animals behavioral analysis before PET/CT was performed. The animals received a dose of FDG via tail vein injection before imaging. FDG, with an activity of $500 \mathrm{Ci} / \mathrm{mmol}$, was prepared. $1.0 \mathrm{mCi}$ of pyrogen-free $18 \mathrm{FDG}$ was injected through the tail vein and the rats were returned to their home cage in a room with minimal ambient noise for the uptake period. Subsequently, the rats were anesthetized with $10 \%$ chloral hydrate $(350 \mathrm{mg} / \mathrm{kg}$; i.p.) by intraperitoneal injection and were placed in the micro PET scanner with their feet extended. No rats displayed signs of peritonitis following chloral hydrate administration. A 15-min static PET acquisition was performed followed by a 5-min anatomical CT scan acquisition. In these conditions, their metabolic rate in the region of interest (ROI) was indirectly assessed to determine the FDG uptake rate, which was quantified as follows: i) Specific uptake values (SUVs) were collected three times across each ROI in the bilateral striatum; and ii) mean SUVs were obtained across each ROI in the striatum (SUV mean). The ROI size was 15-16 pixels and the data were expressed as Becquerel $(\mathrm{Bq})$ /cubic centimeter (CC).

$$
\mathrm{SUV}=\frac{\overline{\mathrm{x}}_{\text {striatum }} \times \mathrm{BW}}{\mathrm{I}_{\text {dose }}}
$$

where $\bar{x}_{\text {striatum }}$ is the mean tracer activity within the ROI of the striatum, $I_{\text {dose }}$ is the injected dose and BW is the body weight. This was accomplished using the PMOD software package (version 2.1; PMOD Technologies LLC) in conjunction with the W. Schiffer rat brain template and atlas (15).

Statistical analysis. The data were analyzed using the SPSS version 24.0 software (IBM Corp.). The normality of data distribution of the continuous variables was assessed using the Shapiro-Wilk test. Continuous variables with normal distribution are presented as the mean \pm standard deviation (SD). The means of normally distributed continuous variables were compared using Tukey's test and one way ANOVA (Respectively, if the results of ANOVA was significant, Tukey's test was used to compare the differences between the two means.). Tukey's test was used to compare between groups and assess variation between timepoints. Correlation between the stereotypy scores and SUVs was assessed using Spearman's rank correlation analysis. $\mathrm{P}<0.05$ was considered to indicate a statistically significant difference. Repeated measures ANOVA was used to analyze stereotypy scores at different time points among the groups.

\section{Results}

Stereotypy scores in a rat model of TD. Stereotypy scores of rats in the experimental group were found to be significantly 


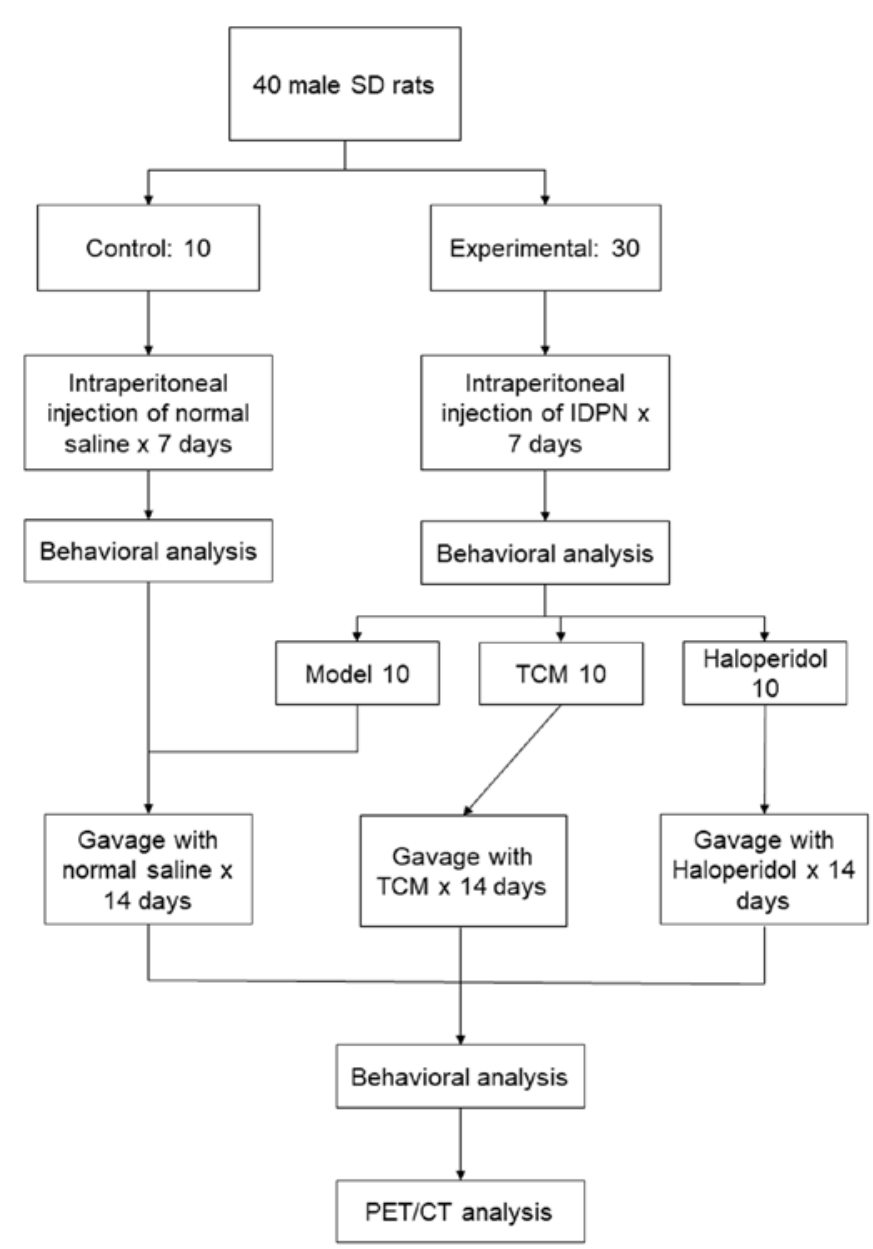

Figure 1. Flow diagram of experimental procedures in SD rats. Male SD rats $(n=40)$ were divided into the control $(n=10)$ and experimental groups $(n=30)$. The experimental group was further divided into the model $(\mathrm{n}=10)$, TCM $(\mathrm{n}=10)$ and the haloperidol groups $(\mathrm{n}=10)$. Behavioral analysis and PET/CT analysis was performed on all rats. SD, Sprague-Dawley; TCM, traditional Chinese medicine; PET/CT, positron emission tomography/CT.

higher compared with those in the control group (repeated measures ANOVA; $\mathrm{P}=0.0005$; Table I). In the first period of observation (5-10 min), the scores of the model group were higher $(3.5 \pm 0.52)$ compared with those in the control group $(0.5 \pm 0.16 ; \mathrm{P}<0.05)$. In the remaining two periods of observation, scores in the model group were also higher (15-20, 3.30 $\pm 0.48 ; 25-30 \mathrm{~min}, 3.31 \pm 0.46)$ compared with those in the control group $(15-20,0.30 \pm 0.28 ; 25-30 \mathrm{~min}, 0.50 \pm 0.40$; $\mathrm{P}<0.05$; Table I).

Stereotypy scores after TCM intervention. After intervention, the scores of rats that received TCM and haloperidol were found to be partially but significantly reduced compared with scores in the model only group (Repeated measures ANOVA; $\mathrm{P}<0.05)$. The magnitude of reduction in stereotypy score in the TCM group $(5-10,1.60 \pm 0.5 ; 10-15 \mathrm{~min}, 1.50 \pm 0.52$; 25-30 min, 1.30 \pm 0.67$)$ was found to be higher compared with that in the haloperidol group $(5-10,2.00 \pm 0.67 ; 10-15 \mathrm{~min}$, $2.20 \pm 0.92 ; 25-30 \mathrm{~min}, 1.80 \pm 0.63$; Table II). The stereotypy scores of TCM group and haloperidol group were significantly lower than that of model only group (both $\mathrm{P}<0.05$ ), while they were still significantly higher than control group $(\mathrm{P}<0.05$; Table II; Fig. 2).

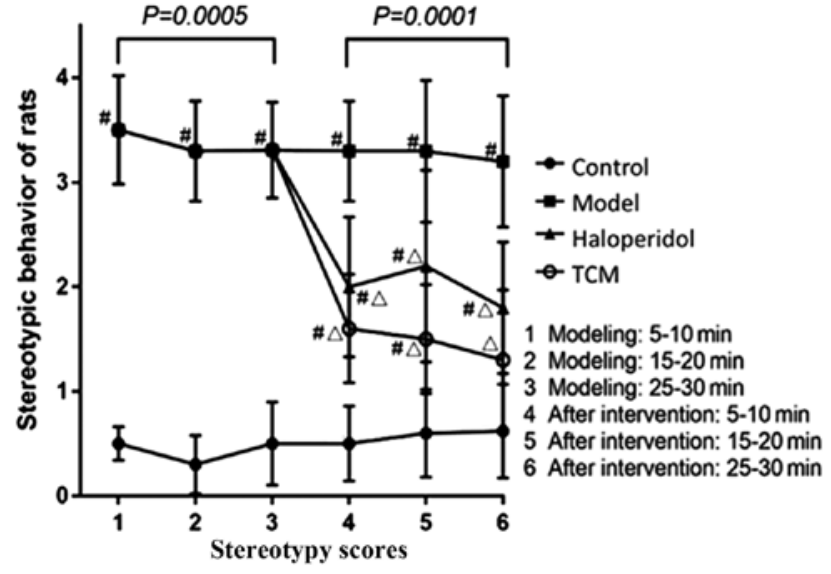

Figure 2. Stereotypy scores after modeling and intervention. After intervention, the scores of rats that received TCM and haloperidol were found to be partially but significantly reduced compared with scores in the model only group (Repeated measures ANOVA; $\mathrm{P}<0.05)$. The magnitude of reduction in stereotypy score in the TCM group $(5-10,1.60 \pm 0.5 ; 10-15,1.50 \pm 0.52$; 25-30 min, 1.30 \pm 0.67$)$ was found to be higher compared with that in the haloperidol group $(5-10,2.00 \pm 0.67 ; 10-15,2.20 \pm 0.92 ; 25-30 \mathrm{~min}, 1.80 \pm 0.63)$. ${ }^{\#} \mathrm{P}<0.05$ vs. control; ${ }^{\Delta} \mathrm{P}<0.05$ vs. model.

SUV of the striatum ROI. FDG PET/CT scans were performed on the rats in the four treatment groups (Fig. 3). The PET signal showed severe hypo-metabolism in the striatum with a marked reduction in FDG uptake in rats from the haloperidol and TCM groups when compared with those in the model only group (control, 1.88 \pm 0.15 ; model only, $3.20 \pm 0.35$; haloperidol, 2.87 \pm 0.18 ; TCM, 2.25 \pm 0.29 ). Tukey's test indicated that FDG uptake in the model group was significantly higher than that of control group $(\mathrm{P}<0.05)$, while FDG uptake in the TCM group was significantly lower than in the model group $(\mathrm{P}<0.05)$.

SUV and stereotypy scores. Correlation between the stereotypy scores after intervention and the SUVs were then analyzed. A moderately positive moderate but significant correlation was identified between the SUVs and the stereotypy scores (Spearman's rank correlation analysis; $\mathrm{R}=0.926$; $\mathrm{P}=0.001$; Fig. 4). One-way ANOVA showed that the differences in the SUVs between each score category was statistically significant $(\mathrm{P}<0.05)$.

\section{Discussion}

Tourette's syndrome (TS) is the most severe form of TD that is a childhood-onset condition and features multiple motor and $\geq 1$ phonic tic lasting $>1$ year (16). In total, $~ 85 \%$ patients with TS suffer from psychiatric comorbidities including, in order of prevalence, attention deficit hyperactivity and obsessive-compulsive disorder, followed by anxiety, mood, autistic spectrum, oppositional defiant and conduct disorders and personality disorders (17). Those disorders may impact patients' academic progress and family relationships of the patients. There are several potential interventions for TDs, including pharmacological treatment (2) and behavioral and psychosocial conditioning (18). However, no universal objective indices currently exist for evaluating the severity of these diseases. In general, the evaluation of tics and comorbidities in children and adults is reliant 

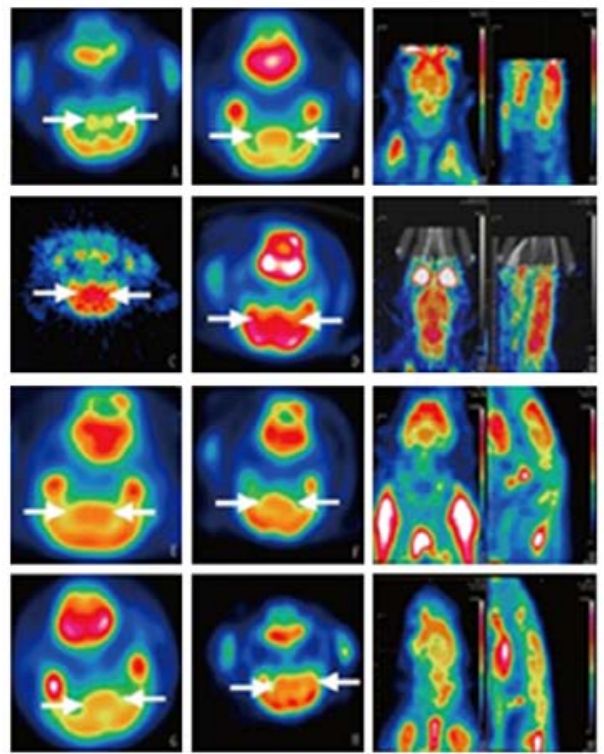

Transverse section
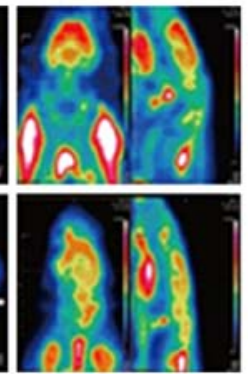

Coronal Sagittal section section

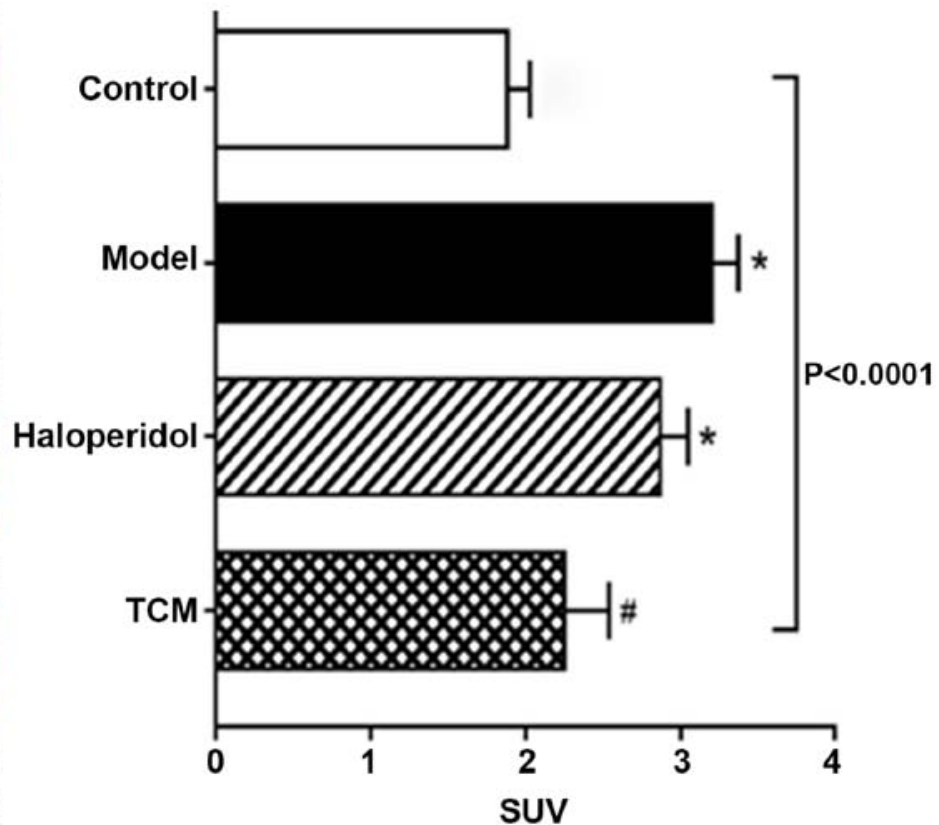

Figure 3. PET signal after intervention. PET images of rats were used to calculate the $18 \mathrm{~F}$-fluorodeoxyglucose tissue uptake rate. The histogram indicates the SUV among the different groups. The arrow points to the region of interest, the striatum. " $\mathrm{P}<0.05$ vs. model only; ${ }^{*} \mathrm{P}<0.05$ vs. control. PET, positron emission tomography; TCM, traditional Chinese medicine; SUV, standardized uptake values.

Table I. Comparison of stereotypy behavior in control and tic disorder model rats.

\begin{tabular}{lcccc}
\hline & & \multicolumn{3}{c}{ Stereotypy score at each timepoint } \\
\cline { 3 - 5 } Group & $\mathrm{N}$ & $5-10 \mathrm{~min}$ & $15-20 \mathrm{~min}$ & $25-30 \mathrm{~min}$ \\
\hline Control & 10 & $0.50 \pm 0.16$ & $0.30 \pm 0.28$ & $0.50 \pm 0.40$ \\
Experimental & 30 & $3.50 \pm 0.52^{\mathrm{a}}$ & $3.30 \pm 0.48^{\mathrm{a}}$ & $3.31 \pm 0.46^{\mathrm{a}}$ \\
\hline
\end{tabular}

aP $<0.05$ vs. control.

Table II. Comparison of stereotypy behavior in control and tic disorder rats after intervention.

\begin{tabular}{lllll}
\hline & & \multicolumn{3}{c}{ Stereotypy scores at each timepoint } \\
\cline { 3 - 5 } Group & $\mathrm{N}$ & $5-10 \mathrm{~min}$ & $15-20 \mathrm{~min}$ & $25-30 \mathrm{~min}$ \\
\hline Control & 10 & $0.50 \pm 0.36$ & $0.60 \pm 0.42$ & $0.62 \pm 0.45$ \\
Model & 10 & $3.30 \pm 0.48^{\mathrm{a}}$ & $3.30 \pm 0.68^{\mathrm{a}}$ & $3.20 \pm 0.63^{\mathrm{a}}$ \\
Haloperidol & 10 & $2.00 \pm 0.67^{\mathrm{a}, \mathrm{b}}$ & $2.20 \pm 0.92^{\mathrm{a}, \mathrm{b}}$ & $1.80 \pm 0.63^{\mathrm{a}, \mathrm{b}}$ \\
TCM & 10 & $1.60 \pm 0.52^{\mathrm{a}, \mathrm{b}}$ & $1.50 \pm 0.52^{\mathrm{a}, \mathrm{b}}$ & $1.30 \pm 0.67^{\mathrm{b}}$ \\
\hline
\end{tabular}

${ }^{\mathrm{a}} \mathrm{P}<0.05$ vs. control, ${ }^{\mathrm{b}} \mathrm{P}<0.05$ vs. model. TCM, traditional Chinese medicine.

on self-reporting scales and clinician-derived interviews. The most widely used checklists of tic characteristics and severity that combine an observation component with historical information obtained from the patients' parents and/or spouses include the YGTSS (19), Shapiro Tourette
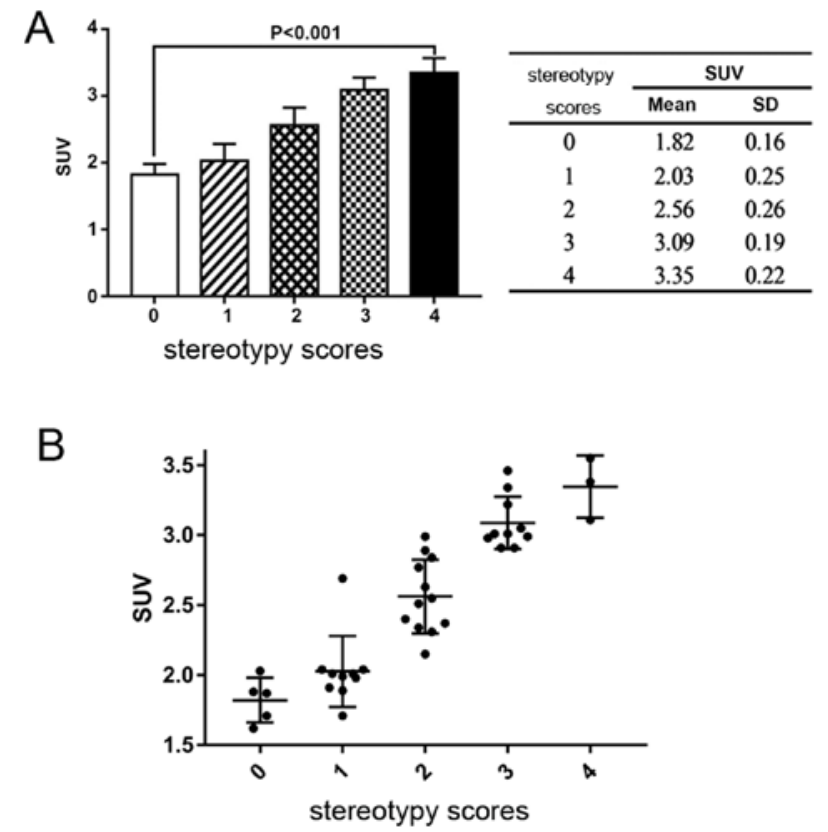

Figure 4. (A) Histogram. Moderately positive moderate but significant correlation was identified between the SUVs and the stereotypy scores; (B) Scatter diagram. ANOVA showed that the differences in the SUVs between each score category was statistically significant.

syndrome severity scale and the Hopkins motor and vocal tic scale (20).

IDPN has been widely used as a tool for neuropathological studies, since it can induce a series of neurobehavioral disturbances, including dyskinesia and repetitive motor-defects, characteristics comparable to that of TS (10). In the present study, IDPN was used to develop a rat model of TD. However, there are limitations to this animal model. The heritability patterns of TS and other tic disorders indicate that the 
pathogenesis of these conditions is also strongly influenced by genetic factors (21). TD animal models based on genetic manipulation have emerged, which may be more accurate compared with drug intervention-induced TD animal models (16).

Abnormalities in the striatum is an emerging topic in studies into TD pathogenesis. Abnormal dopamine uptake in the striatum of TD patients has been reported, where enhanced dopamine uptake within the striatum is believed to be associated with TDs (22). Makki et al (23) previously found that microstructural abnormalities of the striatum in children contribute to the pathophysiology of TS. A transcriptome analysis of the human striatum in TS also suggested that metabolic alterations may be linked to the condition (24). FDG PET/CT is a well-established method for characterizing functional activity in the living human brain. Regional glucose metabolism, coupled with energy-requiring processes driven by the synaptic activity of neurons, appears to be a reliable index of functional activity within the central nervous system (25). The present study suggests that FDG PET/CT can be a useful tool for screening metabolic alterations in the striatum.

An appropriate evaluation indicator would be beneficial in the evaluation of TD treatment. The aim of the present study was to determine the diagnostic performance of FDG PET/CT in the evaluation of TD in rat models. The results of the present study suggested that FDG PET/CT of the striatum can be used to evaluate the effectiveness of TCM and haloperidol treatment for TD in rats, where the SUVs positively correlated with the stereotypy scores. However, the present study has a number of limitations. Since the optimal cutoff values of the mean max SUV were calculated from the sum of the maximum sensitivity and specificity, the performance ability may have been overestimated. Additionally, the number of rats used in the present study was low. This method of evaluation was tested only on a rat model of TD. Further study involving human patients is required.

In summary, the present study suggests that FDG PET/CT of the striatum may be useful for the evaluation of the effectiveness of treatment of TDs. However, further clinical studies are needed to clarify the sensitivity of FDG PET/CT in the evaluation of patients with TD and to investigate the association between metabolic abnormalities and the severity of clinical symptoms.

\section{Acknowledgements}

The authors would like to thank Dr Ming Ruan (Ruijin Hospital affiliated to Shanghai Jiao Tong University School of Medicine) for his help and guidance in the use of Micro PET/CT.

\section{Funding}

The present research was supported by grants from The Shanghai Municipal Commission of Health and Family Planning (grant no. ZYKC201701011) and the National Natural Science Foundation of China (grant no. 8187150436).

\section{Availability of data and materials}

The datasets used and/or analyzed during the current study are available from the corresponding author on reasonable request.

\section{Authors' contributions}

PZ and MW designed the study; PZ, BM and SW performed the experiments; PZ and MW collected the data; PZ and MY analyzed the data; and BM and SW prepared the manuscript. All authors read and approved the final manuscript.

\section{Ethics approval and consent to participate}

All procedures were approved by Xinhua Hospital Affiliated to Shanghai Jiao Tong University School of Medicine (approval no. XHEC-F-2020-017).

\section{Patient consent for publication}

Not applicable.

\section{Competing interests}

The authors declare that they have no competing interests.

\section{References}

1. Steeves T, McKinlay BD, Gorman D, Billinghurst L, Day L, Carroll A, Dion Y, Doja A, Luscombe S, Sandor P and Pringsheim T: Canadian guidelines for the evidence-based treatment of tic disorders: Behavioural therapy, deep brain stimulation, and transcranial magnetic stimulation. Can J Psychiatry 57: 144-151, 2012.

2. Roessner V, Plessen KJ, Rothenberger A, Ludolph AG, Rizzo R, Skov L, Strand G, Stern JS, Termine C and Hoekstra PJ; ESSTS Guidelines Group: European clinical guidelines for Tourette syndrome and other tic disorders. Part II: Pharmacological treatment. Eur Child Adolesc Psychiatry 20: 173-196, 2011.

3. Yang C, Zhang L, Zhu P, Zhu C and Guo Q: The prevalence of tic disorders for children in China: A systematic review and meta-analysis. Medicine 95: e4354, 2016.

4. Abramovitch A, Reese H, Woods DW, Peterson A, Deckersbach T, Piacentini J, Scahill L and Wilhelm S: Psychometric properties of a self-report instrument for the assessment of tic severity in adults with tic disorders. Behav Ther 46: 786-796, 2015.

5. Martino D, Pringsheim TM, Cavanna AE, Colosimo C, Hartmann A, Leckman JF, Luo S, Munchau A, Goetz CG, Stebbins GT, et al: Systematic review of severity scales and screening instruments for tics: Critique and recommendations. Mov Disord 32: 467-473, 2017.

6. Rapanelli M, Frick L, Pogorelov V, Ohtsu H, Bito H and Pittenger C: Histamine H3R receptor activation in the dorsal striatum triggers stereotypies in a mouse model of tic disorders. Transl Psychiatry 7: e1013, 2017.

7. Vinner E, Israelashvili M and Bar-Gad I: Prolonged striatal disinhibition as a chronic animal model of tic disorders. J Neurosci Methods 292: 20-29, 2017.

8. Ding L, Yang Z, Liu G, Ran N, Yi M, Li H, Zhao H, Tang L, Cheng H, Zhao J, et al: Safety and efficacy of taurine as an add-on treatment for tics in youngsters. Eur J Neurol 27: 490-497, 2020.

9. Gerstner W: Time structure of the activity in neural network models. Phys Rev E Stat Phys Plasmas Fluids Relat Interdiscip Topics 51: 738-758, 1995.

10. Zhao L, Qi F, Zhang F, Wang Z, Mu L, Wang Y, En Q, Li J, Du Y and Li A: Dual regulating effect of Ningdong granule on extracellular dopamine content of two kinds of Tourette's syndrome rat models. Biosci Trends 9: 245-251, 2015.

11. Wang Y, Li M, Liang Y, Yang Y, Liu Z, Yao K, Chen Z and Zhai S: Chinese herbal medicine for the treatment of depression: Applications, efficacies and mechanisms. Curr Pharm Des 23: 5180-5190, 2017.

12. Braun AR, Stoetter B, Randolph C, Hsiao JK, Vladar K, Gernert J, Carson RE, Herscovitch P and Chase TN: The functional neuroanatomy of Tourette's syndrome: An FDG-PET study. I. Regional changes in cerebral glucose metabolism differentiating patients and controls. Neuropsychopharmacology 9: 277-291, 1993. 
13. Jeffries KJ, Schooler C, Schoenbach C, Herscovitch P, Chase TN and Braun AR: The functional neuroanatomy of Tourette's syndrome: An FDG PET study III: Functional coupling of regional cerebral metabolic rates. Neuropsychopharmacology 27: 92-104, 2002.

14. Sun LY, Li QP, Zhao LL and Ding YQ: Traditional Chinese medicine inheritance system analysis of professor Ding Yuanqing in treating tic disorder medication based on experience. Zhongguo Zhong Yao Za Zhi 40: 3314-3318, 2015 (In Chinese).

15. Schiffer WK, Mirrione MM, Biegon A, Alexoff DL, Patel V and Dewey SL: Serial microPET measures of the metabolic reaction to a microdialysis probe implant. J Neurosci Methods 155: 272-284, 2006

16. Godar SC, Mosher LJ, Di Giovanni G and Bortolato M: Animal models of tic disorders: A translational perspective. J Neurosci Methods 238: 54-69, 2014.

17. Martino D, Ganos C and Pringsheim TM: Tourette syndrome and chronic Tic disorders: The clinical spectrum beyond Tics. Int Rev Neurobiol 134: 1461-1490, 2017.

18. Verdellen C, van de Griendt J, Hartmann A and Murphy T; ESSTS Guidelines Group: European clinical guidelines for Tourette syndrome and other tic disorders. Part III: Behavioural and psychosocial interventions. Eur Child Adolesc Psychiatry 20: 197-207, 2011.
19. Leckman JF, Riddle MA, Hardin MT, Ort SI, Swartz KL, Stevenson J and Cohen DJ: The Yale Global Tic severity scale: Initial testing of a clinician-rated scale of tic severity. J Am Acad Child Adolesc Psychiatry 28: 566-573, 1989.

20. Walkup JT, Rosenberg LA, Brown J and Singer HS: The validity of instruments measuring tic severity in Tourette's syndrome. J Am Acad Child Adolesc Psychiatry 31: 472-477, 1992.

21. Qi Y, Zheng Y, Li Z, Liu Z and Xiong L: Genetic studies of Tic disorders and Tourette syndrome. Methods Mol Biol 2011: 547-571, 2019.

22. Singer HS, Hahn IH and Moran TH: Abnormal dopamine uptake sites in postmortem striatum from patients with Tourette's syndrome. Ann Neurol 30: 558-562, 1991.

23. Makki MI, Behen M, Bhatt A, Wilson B and Chugani HT: Microstructural abnormalities of striatum and thalamus in children with Tourette syndrome. Mov Disord 23: 2349-2356, 2008.

24. Lennington JB, Coppola G, Kataoka-Sasaki Y, Fernandez TV, Palejev D, Li Y, Huttner A, Pletikos M, Sestan N, Leckman JF and Vaccarino FM: Transcriptome analysis of the human striatum in Tourette syndrome. Biol Psychiatry 79: 372-382, 2016.

25. Chugani HT: Imaging brain metabolism in the newborn. J Child Neurol 33: 851-860, 2018. 